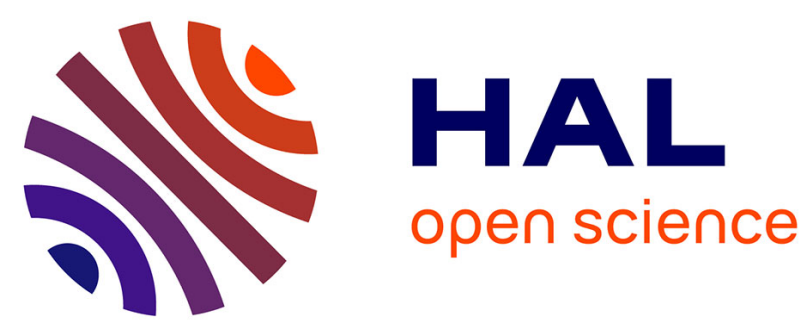

\title{
Kelvin force microscopy characterization of charging effect in thin a-SiOxNy:H layers deposited in pulsed plasma enhanced chemical vapordeposition process by tuning the Silicon-environment
}

Christina Villeneuve-Faure, Kremena Makasheva, Caroline Bonafos, Bernard Despax, Laurent Boudou, Patrick Pons, G. Teyssedre

\section{To cite this version:}

Christina Villeneuve-Faure, Kremena Makasheva, Caroline Bonafos, Bernard Despax, Laurent Boudou, et al.. Kelvin force microscopy characterization of charging effect in thin a-SiOxNy:H layers deposited in pulsed plasma enhanced chemical vapordeposition process by tuning the Siliconenvironment. Journal of Applied Physics, 2013, 113 (20), pp.204102. hal-00878200

\author{
HAL Id: hal-00878200 \\ https://hal.science/hal-00878200
}

Submitted on 29 Oct 2013

HAL is a multi-disciplinary open access archive for the deposit and dissemination of scientific research documents, whether they are published or not. The documents may come from teaching and research institutions in France or abroad, or from public or private research centers.
L'archive ouverte pluridisciplinaire HAL, est destinée au dépôt et à la diffusion de documents scientifiques de niveau recherche, publiés ou non, émanant des établissements d'enseignement et de recherche français ou étrangers, des laboratoires publics ou privés. 


\title{
Kelvin Force Microscopy characterization of charging effect in thin $\mathrm{a}-\mathrm{SiO}_{\mathrm{x}} \mathrm{N}_{\mathrm{y}}: \mathrm{H}$ layers deposited by PPECVD through tuning with the Si-environment
}

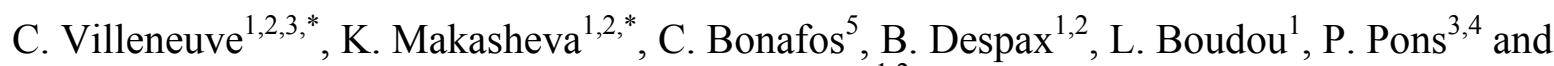 \\ G. Teyssedre ${ }^{1,2}$
}

${ }^{1}$ Université de Toulouse; UPS, INPT; LAPLACE (Laboratoire Plasma et Conversion d'Energie);

118 route de Narbonne, F-31062 Toulouse, France

${ }^{2}$ CNRS; LAPLACE; F-31062 Toulouse, France

${ }^{3}$ CNRS; LAAS; 7 avenue du colonel Roche, F-31077 Toulouse, France

${ }^{4}$ Université de Toulouse; UPS, INSA, INP, ISAE; LAAS; F-31077 Toulouse, France

${ }^{5}$ CEMES-CNRS, Université de Toulouse, 29 rue Jeanne Marvig, F-31055 Toulouse, France

*Corresponding authors: christina.villeneuve@laplace.univ-tlse.fr

kremena.makasheva@laplace.univ-tlse.fr

\begin{abstract}
In this paper, results about tuning of electrical properties of a-SION:H thin film is presented. Issues related to structural and electrical characterization of these layers is investigated. Ellipsometry is used to determine accurately layer thickness and Kelvin Force Microscopy (KFM) permits to characterize local electrical properties. Results emphasize that tuning layer process parameter permit to modify surface and volume conduction. More particularly, increasing of Si-environment increases volume conduction and charges retention. So, local electrical properties of dielectric film can be engineered with this process
\end{abstract}

\section{Acknowledgments}

This work was supported by the RTRA STAE in France under project SYMIAE. The authors acknowledge financial support from the French CNRS and CEA METSA network for the TEM observations. 


\section{Introduction}

Dielectric materials regularly take part of electrical engineering solutions as components on a very large scale: from millimeter to nanometer size. Knowledge of the composition of dielectric materials and their behavior under different kind of stress (electrical, mechanical, etc.) is required to assure the reliability of the insulating parts. The performance of applied dielectric layers is of great significance in order to guarantee the performance of the system as a whole. It is generally acknowledged that the dielectric charging phenomenon is one of the challenges to meet for many electrical systems. Indeed, electrical charging in thin films is present in many important technologies and processes. It is the basis for everyday applications memory ${ }^{1}$, electrets ${ }^{2}$. However, it is equally involved in some dielectric troubleshoots like electrostatic adhesion in MicroElectroMechanical System ${ }^{3}$ (MEMS) or damage in electronic devices ${ }^{4}$ caused by uncontrolled electrostatic discharge. Thus, it represents an obstacle for the system's performance as it modifies the control parameters and in certain cases it can even lead to complete loss of command of these systems.

The electrostatic charging of dielectric materials is a physical phenomenon that raises questions at different levels: (i) scientific level ${ }^{5}$; (ii) diagnostic one ${ }^{6}$ and (iii) technological one [Ref]. The origin of dielectric charging, charge retention and charge evacuation is constantly a subject of scientific research in different insulating materials aiming at to better understand this phenomenon and its behavior in order to control or to avoid the electrostatic charging of dielectric layers [Refs - Gilbert]. Consequently, charge distribution in insulators has received considerable attention but it still signifies great scientific challenge, largely due to a current lack of knowledge on the nature and related specific mechanisms. Moreover, when layer thickness decrease under micron, specific issues are superimposed [5-Rezende]. A literature overview points out that thin film characterization at nanoscale7 level is a scientific and technical challenge. Critical points are focused on the determination of local properties

\footnotetext{
${ }^{1} \mathrm{P}$. Normandaet al. "Effects of annealing conditions on charge storage of Si nanocrystal memory devices obtained by low-energy ion beam synthesis" Microelec. Eng. 67-68, 629 (2003).

${ }^{2}$ Gerhard-Mulhaupt R and Joseph M C 1999 Electrets 3rd edn(California: Laplacian press)

${ }^{3}$ A. Witvrouw, H.A.C. Tilmans and I. De Wolf "Materials issues in the processing, the operation and the reliability of MEMS" Microelectronic Engineering 76, 245 (2004).

${ }^{4}$ Material available from http://www.esda.org/

${ }^{5}$ C. A. Rezende, R. F. Gouveia, M. A. da Silva, and F. Galembeck, "Detection of charge distribution in insulator surfaces", J. Phys.: Condens. Matter, vol. 21, 263002, 2009.

${ }^{6}$ M. Lamhamdi, P. Pons, U. Zaghloul, L. Boudou, F. Coccetti, J. Guastavino, Y. Segui, G. Papaioannou and R. Plana, "Voltage and temperature effect on dielectric charging for RF-MEMS capacitive switches reliability investigation", Microelectronics Reliability, vol. 48, 1248, 2008.

${ }^{7}$ R.K. Leach et al. "The European nanotechnology landscape”, Nanotech 22, 062001 (2011)
} 
(mechanical, electrical...) and in dielectric case mechanism involved in charging phenomenon and charges retention.

To determine charge distribution in thin dielectric films $(<1 \mu \mathrm{m})$ classical diagnostic techniques, like Focused Laser Intensity Modulation Methods which has $1 \mu \mathrm{m}$-resolution, fail. Thus, Scanning Force Mircroscopy (SPM) is naturally used to inject charges and to image the resulting surface potential.

Initially, SPM has been used to inject charges in dielectric materials and in semiconductors $^{8}$ followed by measurements of the resulting potentials by Electrosctatic Force Microscopy ${ }^{9,10,11}$. Furthermore, the stored charge density is quantified by applying this powerful method. However, recent studies provide a nuanced view of the EFM procedure, namely showing its sensitivity to applied bias during measurement and its accuracy limitation induced by imaged charge effects ${ }^{12,13}$. To avoid these adverse effects Kelvin Force Microscopy (KFM), through its ability to characterized charges in dielectric materials, has been applied and demonstrated experimentally [13-Morita, 5-Rezende, and Ishii ${ }^{14}$ ].

From an experimental point of view, some aspects need to be accurately controlled to identify injection and decay phenomena related to materials under study (environmental conditions: no humidity, measurements under $\mathrm{N}_{2}$ environment [5-Rezende, 13-Morita] ${ }^{15}$, lifthigh $^{16}$ ) and to potential impact on topography ${ }^{17}$.

This paper is dedicated to an experimental study of charge injection and charge decay in thin dielectric amorphous silicon oxy-nitride layers $\left(\mathrm{a}-\mathrm{SiO}_{\mathrm{x}} \mathrm{N}_{\mathrm{y}}: \mathrm{H}\right)$. The layers are deposited by Pulsed Plasma Enhanced Chemical Vapor Deposition (PPECVD). The PPECVD technique

\footnotetext{
${ }^{8}$ J.E. Stern, B.D.Terris, H.J. Mamin and D. Rugar "Deposition and imaging of localized charge on insulator surfaces using force microscope", A. Phys. Lett. 53, 2717 (1988)

${ }^{9}$ F. Marchi, R. Dianoux, H.J.H. Smilde, P. Mur, F. Comin, J. Chevrier "Characterisation of trapped electric charge carriers behaviour at nanometer scale by electrostatic force microscopy" Journal of Electrostatics 66, 538 (2008)

${ }^{10}$ J. Lambert, G. de Loubens, C. Guthmann and M. Saint-Jean « Dispersive charge transport along the surface of an insulating layer observed by electrostatic force microscopy" Phys. Rev. B 71, 155418 (2005).

${ }^{11}$ S.-D. Tzeng and S. Gwo" Charge trapping properties at silicon nitride/silicon oxide interface studied by variable-temperature electrostatic force microscopy”. Appl. Phys. 100, 023711 (2006).

${ }^{12}$ Lambert 2003

${ }^{13}$ S. Morita, T. Uchihashi, K. Okamoto, M. Abe and Y. Sugawara Microscale contact charging on silicon oxide“ "Scanning probe microscopy : characterization, nanofabrication and Dev. Appl. 289-308 (2005)

${ }^{14} \mathrm{M}$. Ishii, "Static states and dynamic behaviour of charges: observation and control by scanning probe microscopy" J. Phys. Cond. Matter 22, 173001 (2010).

${ }^{15}$ U. Zaghloul, B. Bushan, P. Pons, G.J. Papaioannou, F. Coccetti and R. Plana “ On the influence of environment gases, relative humidity and gas purification on dielectric charging/discharging processes in electrostatically driven MEMS/NEMS devices", Nanotech 22, 035705 (2011).

${ }^{16}$ U. Zaghloul, G. Papaioannou, F. Coccetti, P. Pons and R. Plana " A systematic reliability investigation of the dielectric charging process in electrostatically actuated MEMS based on Kelvin Force Microscopy”, J. Micromech. Microeng.20, 064016 (2010).

${ }^{17}$ D. Ziegler, N. Naujoksans A. Stemmer "Feed-forward compensation of surface potential in atomic force microscopy", Review of Sc. Instrum 79, 063704 (2208)
} 
permits a flexible elaboration of the layers through fine tuning of their electrical properties closely linked to their structural properties. Charge behavior and evolution in the dielectric layer are followed by Kelvin Probe Microscopy.

The paper is organized as follow: the experimental details are presented in section II. It is divided in three sub-sections representing respectively the deposition and the diagnostic methods for structural and for the electrical characterization. The obtained results are shown in section III. They are accompanied by a discussion on the properties and the performance of the deposited layers. Finally, section IV summarizes the main conclusions.

\section{Experimental part}

\section{a. Dielectric layer deposition process}

Thin sub-stoichiometric amorphous silicon oxy-nitride layers $\left(a-\mathrm{SiO}_{\mathrm{x}} \mathrm{N}_{\mathrm{y}}: \mathrm{H}, \mathrm{x}<2, \mathrm{y}<1\right)$ are deposited in the plasma sustained by a radiofrequency (RF) capacitively coupled discharge at frequency of $f=13.56 \mathrm{MHz}$ with power of $5 \mathrm{~W}^{18} 19$. The RF discharge is modulated with square pulses of period $\mathrm{T}=400 \mathrm{~ms}$ and pulse width of $100 \mathrm{~ms}$. The gas mixture contains $\mathrm{SiH}_{4}, \mathrm{~N}_{2} \mathrm{O}$ and He. The ratio $\gamma=\left[\mathrm{N}_{2} \mathrm{O}\right] /\left[\mathrm{SiH}_{4}\right]$ allows adjusting the silicon concentration in the film. The total gas pressure used for deposition is $p=65 \mathrm{~Pa}$. The total gas flow is around $210 \mathrm{sccm}$ (sccm stands for standard cubic centimeters per minute) depending on the gas mixture ( $\gamma$-value). He-component in the mixtures is fixed to $40 \%$. The substrate temperature is kept to $300^{\circ} \mathrm{C}$ during the deposition process.

Dielectric layers with different $\gamma$ values $(\gamma=100,10$ and 5) are deposited on $\mathrm{Au} / \mathrm{Ti}$ metallized Si (100) 4"-wafers. The Ti-layer is used to assure adhesion between the Au-layer and the Si-substrate. High $\gamma$-value $(\gamma=100)$ means that the dielectric layer has properties close to a $\mathrm{SiO}_{2}$ layer. The lower the $\gamma$-value, the richer in $\mathrm{Si}$ the dielectric layer is. As the dielectric layer deposition is preceded by metallization of the Si-substrates the state of the Aulayer is controlled before and after deposition of the a- $\mathrm{SiO}_{\mathrm{x}} \mathrm{N}_{\mathrm{y}}: \mathrm{H}$ layers. Preliminary study on the structural modifications of the Au-layer induced by the dielectric layer deposition procedure, mainly due to the high substrate temperature during the deposition process, is also

\footnotetext{
${ }^{18}$ S. Perret-Tran-Van, K. Makasheva, B. Despax, C. Bonafos, P.E. Coulon, and V. Paillard, "Controlled fabrication of Si-nanocrystals embedded in thin SiON layers by PPECVD followed by oxidizing annealing", Nanotechnology, Vol. 21, 285605 (9pp.), 2010.

${ }^{19}$ K. Makasheva, B. Despax, L. Boudou, and G. Teyssedre, "Dielectric Layers for RF-MEMS Switches: Design and Study of Appropriate Structures Preventing Electrostatic Charging" IEEE Trans. Dielectrics and Electrical Insulation (2012), in press.
} 
performed. Results from that study are used to develop a procedure for spectroscopic ellipsometry (SE) diagnostics for non-destructive characterization of the a- $\mathrm{SiO}_{\mathrm{x}} \mathrm{N}_{\mathrm{y}}: \mathrm{H}$ layers.

\section{b. Structural characterization}

This part is dedicated to layer characterization methods. The layer thickness is determined by spectroscopic ellipsometry measurements. SOPRA GES-5 spectrometer in the spectral range $250-850 \mathrm{~nm}$ has been used. Since the Si-substrates are Au-metallized the incidence angle, which is the most appropriate one to perform the measurements is determined first. We have found that for our Au-metallized Si-wafers the most sensitive and accurate measurements should be performed in the range $68^{\circ}-70^{\circ}$ of the incidence angle. Our choice is $69^{\circ}$. Detailed description of the assessment procedure is given in Appendix 1.

The simulation of the recorded spectra is performed with Winelli ${ }^{20}$, software provided by SOPRA LAB. For spectra processing we have applied Forouhi-Bloomer dispersion law ${ }^{21}$. This approximation provides information on the layer thickness and the optical properties ( $\mathrm{n}-$ refractive index and $\mathrm{k}$ - extinction coefficient) of the layers.

Results concerning layer thickness, composition of layers, state of the interfaces between different layers and possible Au-atom migration in the dielectric layers are critical and essential to improve understanding charging phenomenon in thin dielectric films. The only diagnostic technique that gives possibility to observe the as-deposited layer structures is Transmission Electron Microscopy (TEM) ${ }^{22}$ in a cross sectional view. To achieve observation specimens transparent to electrons have been prepared in accordance with the standard procedure: mechanical polishing and $\mathrm{Ar}^{+}$ion milling. The observations have been performed on PHILIPS CM30 TEM in Bright field mode.

\section{c. KFM setup}

Charge injection and charge decay are studied by Atomic Force Microscopy (AFM) in Kelvin Force Microscopy (KFM) configuration. Specific methodology is developed in order to improve results accuracy and reproducibility. Surface potential measurements and charge injection are performed by using ICON Bruker AFM with PtIr coated probes.

\footnotetext{
${ }^{20}$ WinElli Software by SOPRA S.A., Version 3.04, 1999.

${ }^{21}$ A. R. Forouhi and I. Bloomer, "Optical dispersion relations for amorphous semiconductors and amorphous dielectrics”, Phys. Rev. B, Vol. 34, pp.7018-7026, 1986.

${ }^{22}$ Caroline - Ref
} 
As shown previously ${ }^{23}$ humidity is the most important parameter to be controlled during the study on charge injection and charge transport in dielectric thin layers. To avoid humidity, samples are annealed $5 \mathrm{~min}$ at $120^{\circ} \mathrm{C}$ on hot plate and put in small AFM-cell under $\mathrm{N}_{2}$ flow. Force Distance Curve is used to follow the adhesion force due to water adsorbed on sample surface. When this force becomes equivalent to Van der Walls interaction, humidity influence can be neglected. The same protocol is respected to each studied thin layer.

Charge injection is performed in contact mode with DC voltage (10-25V) during fixed times (10s up to 10min). During injection, the contact force is kept constant at small level $(10 \mathrm{nN})$ in order to respect tip integrity and to obtain reproducible contact. Potential measurements are performed in lift configuration with small lift distance $(5 \mathrm{~nm})$ which is permitted by the small sample roughness.

Comparison between topography figure 1(a) and potential distribution (figure 1(b)) shows that surface is not modified by electrical charges (no link between topography and potential as shown by Ziegler [17-Ziegler]). Horizontal cross-section of the potential profile exhibits Gaussian shape (figure 2(c)) and can be fitted by:

$$
V(x)=V_{0}+\operatorname{Max} \exp \left(-\frac{\left(x-x_{c}\right)^{2}}{2 w^{2}}\right),
$$

where $x_{\mathrm{c}}$ is the lateral position of peak maximum, $w$ is the peak broadening, $V_{0}$ represents the reference surface potential without charges and Max being the maximum difference between $V_{0}$ and the center of charge spot.

To analyze KFM results, four parameters are used: $V_{0}$ - reference surface potential without charges, Max - maximum difference between $V_{0}$ and the center of charge spot, w which is linked to FWHM - the Full Width at Half Maximum of charge distribution $(\mathrm{FWHM}=2 w \sqrt{2 \ln (2)})$ and $I$ - the integrated peak intensity. Moreover, to emphasize decay or injection behavior independently of conditions, normalized integrated intensity $I_{\mathrm{n}}$ and normalized maximum $\operatorname{Max}_{\mathrm{n}}$ are defined. Normalization is done relative to value at reference time: injection time of $10 \mathrm{~s}$ and 30 s after injection for decay.

Injection and KFM measurements reproducibility have been studied and a 5\%standard dispersion around mean value is observed for each parameter of interest.

\footnotetext{
${ }^{23}$ Usama nanotech
} 
In order to provide complementary information, injection current was measured in TUNA mode. This low noise mode, permits to measure very small current (about 30pA) and consequently to follow current during injection.

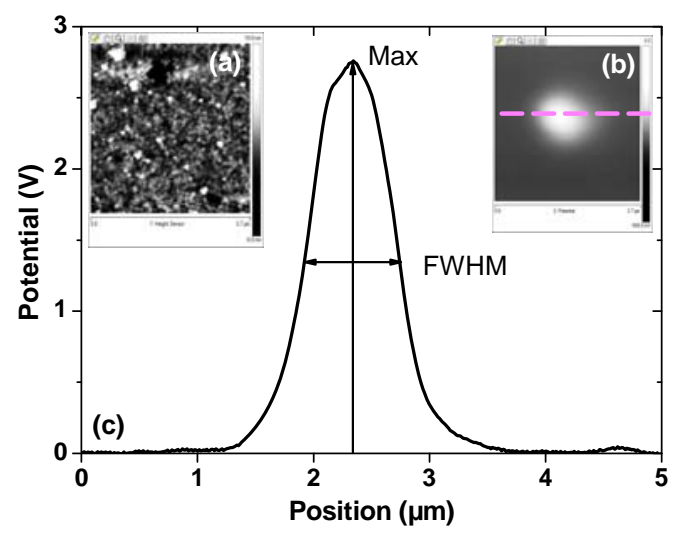

Figure 1: (a) a-SiO $\mathrm{S}_{\mathrm{y}}: \mathrm{H}$ surface topography after injection, (b) potential distribution over a$\mathrm{SiO}_{\mathrm{x}} \mathrm{N}_{\mathrm{y}}: \mathrm{H}$ surface, (c) cross-section profile of $\mathrm{a}-\mathrm{SiO}_{\mathrm{x}} \mathrm{N}_{\mathrm{y}}: \mathrm{H}(\gamma=100)$ surface potential after injection at $10 \mathrm{~V}$ during $2 \mathrm{~min}$. Maximum and FWHM are defined.

\section{Results and discussions}

Results for the layer thickness, the refractive index at $\lambda=632.8 \mathrm{~nm}$ and the optical band gap deduced from Tauc's law of three different a- $\mathrm{SiO}_{\mathrm{x}} \mathrm{N}_{\mathrm{y}}: \mathrm{H}$ layers as obtained from SE measurements are shown in Table 1. The same layers are used for the KFM measurements.

\begin{tabular}{|c|c|c|c|}
\hline $\begin{array}{c}\text { a-SiO } \\
\text { layer }\end{array}$ & Thickness & $\begin{array}{c}\text { Refractive index- } \mathrm{n} \\
\text { (at } \lambda=632.8 \mathrm{~nm})\end{array}$ & Optical band gap - Eg \\
\hline$\gamma=100$ & $116.6 \pm 0.7 \mathrm{~nm}$ & 1.49 & $5.03 \mathrm{eV}$ \\
\hline$\gamma=10$ & $207.0 \pm 6.3 \mathrm{~nm}$ & 1.55 & $4.2 \mathrm{eV}$ \\
\hline$\gamma=5$ & $165.7 \pm 4.6 \mathrm{~nm}$ & 1.61 & $2.8 \mathrm{eV}$ \\
\hline
\end{tabular}

Table 1: Thicknesses and optical properties of the $\mathrm{a}-\mathrm{SiO}_{\mathrm{x}} \mathrm{N}_{\mathrm{y}}: \mathrm{H}$ layers

One can notice that the $\gamma=100 \mathrm{a}-\mathrm{SiO}_{\mathrm{x}} \mathrm{N}_{\mathrm{y}}: \mathrm{H}$ layer has a refractive index close to that of fused silica layer $\left(\mathrm{n}=1.457^{24}\right)$. It is well known that layers deposited by plasma assisted processes are not stoichiometric, hence such a discrepancy in the refractive index of $\gamma=100$ layer compared to that of fused silica layer is not surprising. Moreover, X-ray photoemission spectroscopy measurements of the $\gamma=100$ layer [19-Makasheva] show that the Si2p photoelectron peak includes only the two highest oxidation states: $\mathrm{Si}-\left(\mathrm{Si}-\mathrm{O}_{3}\right)$ and $\mathrm{Si}-\mathrm{O}_{4}$

\footnotetext{
${ }^{24}$ I. H. Malitson, "Interspecimen Comparison of the Refractive Index of Fused Silica", JOSA, 55, 1205, (1965).
} 
tetrahedras. The detected elements in the $\gamma=100$ layer are $\mathrm{Si}, \mathrm{O}$ and $\mathrm{N}$. The N-content in the layer is small (only 4 at. \%). This additional information explains as why the refractive index of $\gamma=100$-layer is slightly higher compared to that of silica layer. The optical band gap of $\gamma=100$ a-SiO $\mathrm{N}_{\mathrm{y}}: \mathrm{H}$ layer, as obtained from Tauc's law, is quite high which defines this layer as dielectric one. The obtained band gap value corresponds to the low limit of spectral range $(\lambda=250 \mathrm{~nm})$ for the used spectrometer in SE measurements. In such case the accuracy in the application of Tauc's law is lowered. It means that in reality the optical band gap of $\gamma=100$ layer can be even higher. However, from structural point of view this layer $(\gamma=100)$ clearly presents insulating properties and can be considered as a good dielectric layer.

The two other layers $(\gamma=10$ and $\gamma=5)$ have higher refractive indexes and lower optical gaps, respectively. These characteristics are closely related to the $\mathrm{Si}$-contents in the layers mainly through the Si-environment in the $\mathrm{Si}-\left(\mathrm{Si}_{4-\mathrm{m}}-\mathrm{O}_{\mathrm{m}}\right)$ tetrahedras with $\mathrm{m}=0-4$. The structural characterization of $\gamma=5$ layer shows that with an optical band gap of only $2.8 \mathrm{eV}$ this layer can represent semi-conducting properties determined by the Si-content. The intermediate position of $\gamma=10$ a- $\mathrm{SiO}_{\mathrm{x}} \mathrm{N}_{\mathrm{y}}: \mathrm{H}$ layer is quite unclear regarding the conducting properties of this layer and possible behaviour under stress, and prevent us from any predictions.

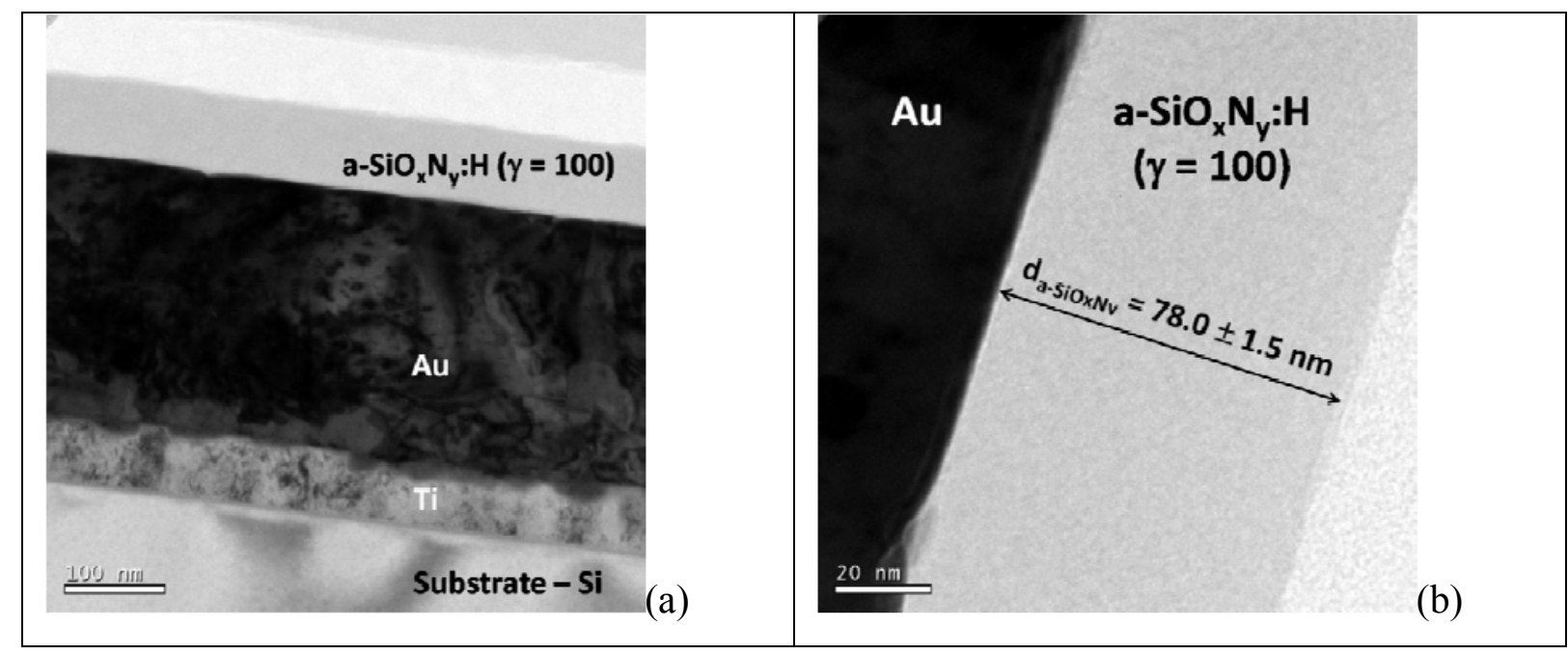

Figure 2: Bright field TEM images of (a) an example of the studied structure: Si-substrate, Tilayer $\left(d_{\mathrm{Ti}}=70 \mathrm{~nm}\right)$, Au-layer $\left(d_{\mathrm{Au}}=260 \mathrm{~nm}\right)$ and the a-SiO $\mathrm{N}_{\mathrm{y}}: \mathrm{H}$ layer $(\gamma=100, d=78.0 \mathrm{~nm})$ and (b) closer view of the $\mathrm{a}-\mathrm{SiO}_{\mathrm{x}} \mathrm{N}_{\mathrm{y}}: \mathrm{H}$ layer $(\gamma=100)$.

As mentioned above state of the interfaces between $\mathrm{Au}$ and $\mathrm{a}-\mathrm{SiO}_{\mathrm{x}} \mathrm{N}_{\mathrm{y}}: \mathrm{H}$ layers and potential Au-atom migration in the dielectric layers are crucial to better understand the 
charging phenomenon in thin dielectric films. The TEM observations in cross-sectional view are indispensable for this purpose. Results depicted on figure 2 show that there is no Au-atom migration in the a-SiO $\mathrm{N}_{\mathrm{y}}: \mathrm{H}$ layers. They reveal that although the applied substrate temperature of $300^{\circ} \mathrm{C}$ during plasma deposition process is close to the $\mathrm{Au} / \mathrm{Si}$ eutectic temperature $\left(363 \pm 2{ }^{\circ} \mathrm{C}^{25}\right)$ it does not imply diffusion of $\mathrm{Au}$ atoms. Moreover, the TEM observations permit to measure layer thickness and results are compared afterwards with results from SE measurements in order to confirm the developed SE procedure. The layer thickness obtained from SE measurements on the sample as shown in figure 1(b) is $d=84.0 \pm 1.5 \mathrm{~nm}$ which is in a very good agreement with the TEM obtained value $(d=78.0 \pm 1.5 \mathrm{~nm})$ for the same sample.

\section{a. Charge Injection}

Charge injection is performed for DC voltages from $10 \mathrm{~V}$ to $25 \mathrm{~V}$ during different time intervals from $10 \mathrm{~s}$ up to $10 \mathrm{~min}$. Electric fields applied to each layer are summarized in Table 2. One can notice that the applied electric fields are very high due to weak layer thickness.

\begin{tabular}{|c|c|c|c|}
\hline \multirow{2}{*}{$\begin{array}{c}\mathrm{a}-\mathrm{SiO}_{\mathrm{x}} \mathrm{N}_{\mathrm{y}}: \mathrm{H} \\
\text { layer }\end{array}$} & Thickness & \multicolumn{2}{|c|}{ Electric field (V/m) } \\
\cline { 3 - 4 } & & $10 \mathrm{~V}$ & $25 \mathrm{~V}$ \\
\hline$\gamma=100$ & $116.6 \pm 0.7 \mathrm{~nm}$ & $8.6 \times 10^{7}$ & $2.1 \times 10^{8}$ \\
\hline$\gamma=10$ & $207.0 \pm 6.3 \mathrm{~nm}$ & $4.8 \times 10^{7}$ & $1.2 \times 10^{8}$ \\
\hline$\gamma=5$ & $165.7 \pm 4.6 \mathrm{~nm}$ & $6.0 \times 10^{7}$ & $1.5 \times 10^{8}$ \\
\hline
\end{tabular}

Table 2: Applied electric fields during charge injection.

A comparison of the resulting charge-profiles, shown in figure 3, exhibits strong difference between the different layers. For $\gamma=100$ and $\gamma=5$ charges are stored closely to injection contact area, whereas $\gamma=10$ demonstrates strong lateral charge spreading. To accurately understand this behavior integral peak intensity $I$ and peak broadening $\Delta w$, for each layer, are studied and compared as function of the injection condition.

Results concerning charge injection, in each layer, are compared in figure 4 and 5. Charging dynamic (normalized integrated intensity $I_{\mathrm{n}}$ ) and linear charge density (integrated intensity $I$ ) are compared for $10 \mathrm{~V}$ and $25 \mathrm{~V}$ injection bias in figure 4 . Whereas, figure 5 , is

\footnotetext{
${ }^{25}$ R. P. Anantatmula, A. A. Johnson, S. P. Gupta and R. J. Horylev, “The gold-silicon phase diagram” J. Electronic Materials, 4, 445, (1975)
} 
dedicated to peak broadening $\Delta w$ with time injection. Regardless of the applied DC voltage, the storage charge density strongly increases with time injection and its dynamic is closely related to the layer structure. No injection threshold is observed, and the injection voltage has no influence on the peak broadening during injection (figure 5). Moreover, the same results are obtained for negative bias (not shown here).

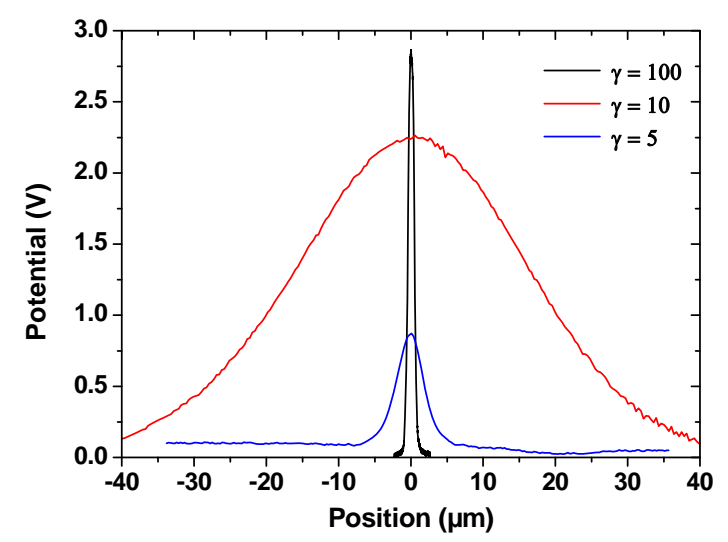

Figure 3: Charge profiles for different layers induced by injection at $25 \mathrm{~V}$ during $2 \mathrm{~min}$.

Concerning $\gamma=100$ layer, only few charges are stored after injection (figure 4). The charges are located close to the contact area $(\mathrm{FWHM}=500 \mathrm{~nm})$ and a peak broadening less than $20 \%$ is observed with time injection increasing (figure 4 ). This is the sign of weak lateral charge diffusion on the layer surface. For high injection voltage, when time injection increase, saturation is observed on injected charge density. It can be concluded that the charge injection is limited by the deep traps available.

Data depicted on figures 4 and 5 show that $\gamma=10$ layer exhibits very different behavior compared to $\gamma=100$ layer. The obtained results emphasize that high charge density is stored in this layer without saturation effect when injection voltage increase (figure 4). Moreover, the spot broadening is important $(\mathrm{FWHM}=20 \mu \mathrm{m})$ and strongly influenced by injection time (figure 5), with FWHM increasing up to $70 \%$ for $10 \mathrm{~min}$ injection. This strong spreading around contact area, points out important charges diffusion on the surface. For $\gamma=10$-layers with more important thickness $(350 \mathrm{~nm})$ the same effect is observed: high charge density is stored and without saturation effect when injection time increases. However, the peak broadening is weaker than lower thickness. So, surface diffusion is ruled by layer thickness and consequently by phenomenon which occurred in volume. These effects seem to be linked with the deep traps located under the AFM tip which are actually induced by the 
high electric field. The created space charge prevents charge conduction in the volume and promotes occupation of lateral shallow traps. Therefore, when the layer thickness decreases, charge blocking in the layer volume occurs earlier and the peak broadening is stronger.

Quick look at the obtained results discloses that $\gamma=5$-layer represents remarkable behavior. Indeed, in this layer, only few charges are stored and they are localized close to contact area $(\mathrm{FWHM}=5 \mu \mathrm{m})$. Moreover, peak broadening with increasing injection time is important related to initial FWHM (up to 60\%). Figure 4(b) shows that, in this case, charging dynamic is strongly influenced by injection voltage. For high injection voltage, when the injection time increases, a saturation of the injected charge density is observed, whereas no saturation effect appears at low injection voltage. Indeed, for low injection voltage (figure 4(a)), charge injection dynamic is the same for the layers with $\gamma=5$ and $\gamma=10$. On a contrary for high injection voltage (figure 4(b)), the charge injection dynamic of $\gamma=5$-layer follows the same behavior as $\gamma=100$-layer. This observation suggests that two different phenomena occur and manage charge injection in the $\gamma=5$-layer.

Induced current trough the layers during charge injection is followed by TUNA mode. For $\gamma=100$-layer, no current is detected up to $40 \mathrm{~V}$ of DC voltage. This high voltage threshold confirms that in this case, charge injection is limited by the available deep traps. For $\gamma=5$ layer, currents of $50 \mathrm{pA}$ and $150 \mathrm{pA}$ are measured for $10 \mathrm{~V}$ and $25 \mathrm{~V}$ of the injection voltage (results not shown here). These results reveal the fact that, for $\gamma=5$-layer, during charges injection, it is the charge flow through the volume which limits the charging effect. For lower voltages, current flow is smaller, so the injection dynamic is quicker. The same behavior is found for $\gamma=10$ which has high charges storage capability. For high injection voltages, injection dynamic is strongly limited by volume conduction. Consequently, the charge storage is limited and saturation effect is observed. Moreover, current saturation is observed at very high electric fields $\left(>10^{8} \mathrm{~V} / \mathrm{m}^{2}\right)$ which are representative of electron mobility saturation in case of hopping conduction. It's difficult to compare the current results to previously obtained ones by charging/discharging current on MIM structures ${ }^{26}$ as the applied electric fields in the current study are an order of magnitude higher and the layer response is most probably nonlinear. Indeed, current measurements on MIM structures up to these very high fields $\left(>10^{8} \mathrm{~V} / \mathrm{m}^{2}\right)$ failed, leading to a breakdown of the dielectric layer.

To conclude, each layer has very different behavior toward charge storage. $\gamma=100$-layer permits to store only few charges which are strongly localized. In this case, charges storage is

\footnotetext{
${ }^{26}$ K. Makasheva, B. Despax, L. Boudou and G. Teyssedre“ Dielectric layers for RF-MEMS switches : design and study of appropriate structures preventing electrostatic charging" XXX.
} 
limited by deep traps available. $\gamma=10$ layer exhibits important stored charges due to strong surface conduction during injection. This is due to space charge which stops volume conduction. $\gamma=5$ permits to store small charge density because charging mechanism is limited by volume conduction. These differences in charge injection mechanisms foreshadow very different discharging behavior as describe in the following part.

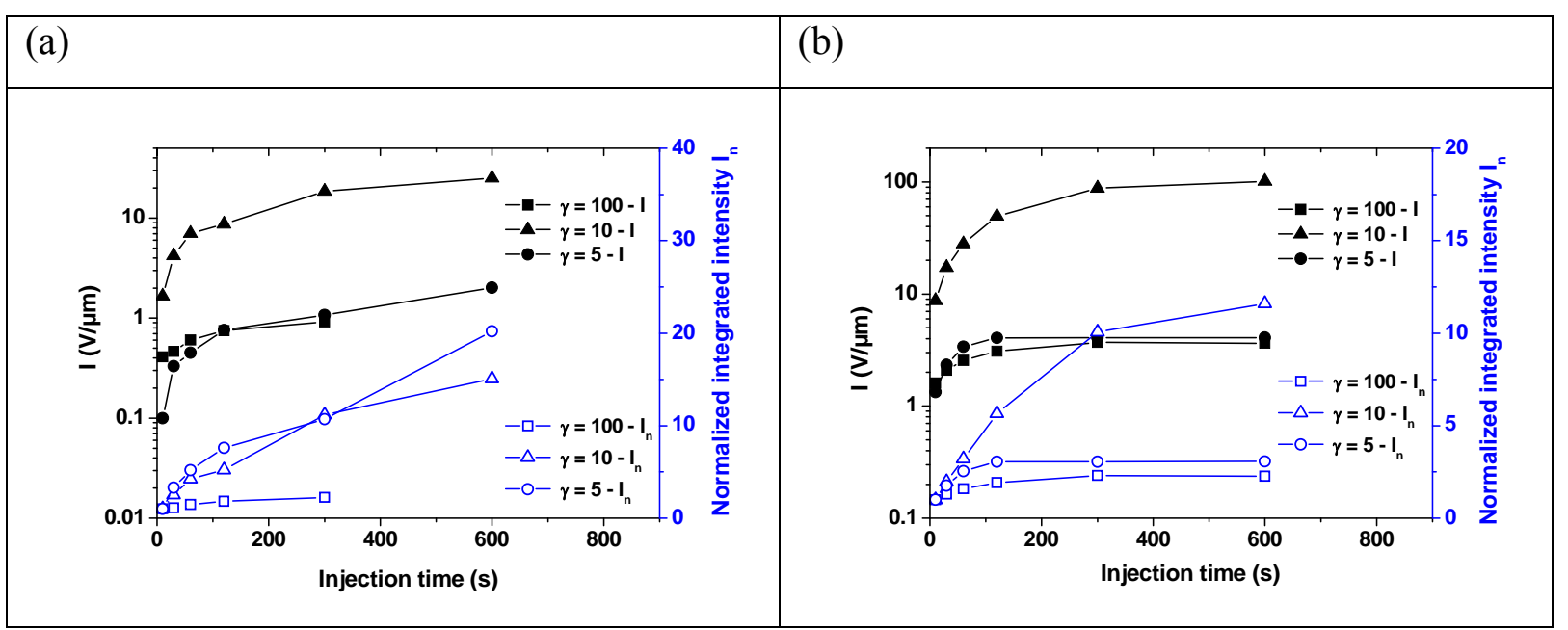

Figure 4: Comparison of evolution of integrated intensity $I$ and normalized integrated intensity $I_{\mathrm{n}}$ with injection time for different a-SiO $\mathrm{x}_{\mathrm{y}}: \mathrm{H}$ layers. Bias injection is fixed to (a) $10 \mathrm{~V}$ and (b) $25 \mathrm{~V}$.

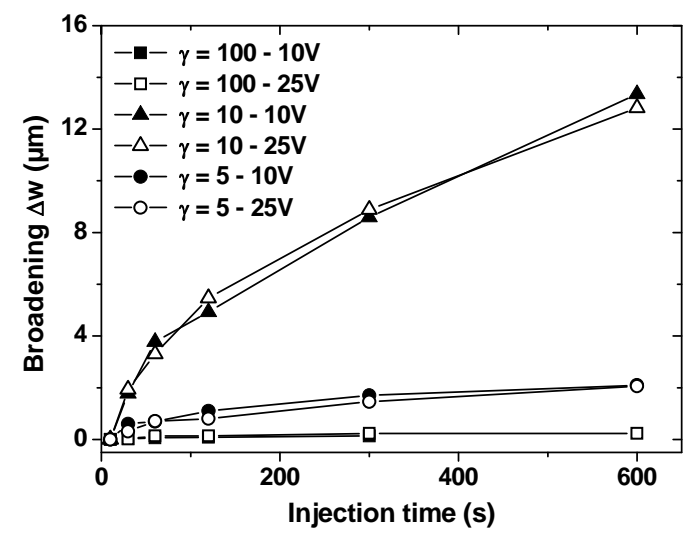

Figure 5: Comparison of evolution of broadening $\Delta \mathrm{w}$ with injection time, for different $\gamma$ values. Bias injection is fixed to $10 \mathrm{~V}$ or to $25 \mathrm{~V}$.

\section{b. Charge transport}

When charges are stored in an insulating material, two dissipation processes are possible: 
- Surface diffusion, which is a conservative mechanism for the charges density. Thus, the integrated intensity $\mathrm{I}$ is constant with time irrespective to potential maximum decrease. Moreover, strong FWHM broadening is observed.

- Volume process (recombination, hopping conduction, etc...), which is a non-conservative mechanism for the charges density. In this case, the integrated intensity $I$ and the maximum potential Max, decrease with the same velocity.

When the two phenomena (surface diffusion and volume process) are superimposed, i.e. act simultaneously, the integrated intensity $I$ and the maximum potential Max decrease with different time constants.

To analyze charge transport after injection, in each layer, the potential maximum Max, the integrated intensity $I$ and the potential profile broadening are studied accurately. To emphasize dynamic, only normalized values are plotted as function of time on figure 6 . Measurements performed for different injection times (not shown here) demonstrate that the injection time has no influence on charge transport dynamic. Thus, only results for charge injection at voltages of $10 \mathrm{~V}$ and $25 \mathrm{~V}$ during $2 \mathrm{~min}$, are reported here.

Concerning $\gamma=100$ layer, integrated intensity is constant whereas slow maximum potential decrease is observed (figure 6(a)). In the same time only small peak broadening occurs. So, charge density is constant and only slow surface diffusion is observed (lateral speed $7 \times 10^{-2} \mathrm{~nm} / \mathrm{s}$ )

As far as the $\gamma=10$ layer is concerned the integrated intensity decreases linearly with time after injection. In the same time the potential maximum decreases exponentially and peak broads nonlinearly (figure 6(b)). This reveals the simultaneous acting of both processes surface diffusion and volume process. At early times after injection $(\mathrm{t}<250 \mathrm{~s})$, the peak broadening is important and the FWHM increases linearly with $60 \mathrm{~nm} / \mathrm{s}$ lateral speed. At long time scale $(\mathrm{t}>250 \mathrm{~s})$, the peak broadening is lowered. The integrated intensity and the potential maximum decrease with same velocity, suggesting that the volume mechanism is predominant. Moreover, as integrated intensity decreases linearly, volume mechanism is invariant with time (charge density decreases with speed of $0.7 \mathrm{Vnm} / \mathrm{s}$ ).

Concerning $\gamma=5$, the integrated intensity, the maximum potential and the broadening evolution with time exhibit two different behavior (figure 6(c)). At early times $(\mathrm{t}<200 \mathrm{~s})$, peak broadening is important and increases linearly with $8 \mathrm{~nm} / \mathrm{s}$ lateral speed. Integrated intensity and potential maximum decrease rapidly, with quite the same speed. Consequently, the volume and the surface mechanisms occur at the same time with predominant volume mechanism. Charge density decreases with high speed $8 \mathrm{Vnm} / \mathrm{s}$ indicating that a fast volume 
mechanism is involved. At long time scale ( $\mathrm{t}>200 \mathrm{~s})$, the peak broadening is negligible. The integrated intensity and the potential maximum decrease slowly with same speed. It suggests that the leading mechanism is a volume one. The charge density decreases with small velocity of only $0.5 \mathrm{Vnm} / \mathrm{s}$ slow volume mechanism is involved.

Analyzing the above presented results one can say that $\gamma=5$ exhibits two different discharging volume dynamics: a high speed mechanism and a low speed one. As the potential maximum decreases with quite the same speed in both $\gamma=10$ and in $\gamma=5$ layers for slow mechanism, it is possible that the same mechanism for decay is involved. However, the KFM measurements cannot give sufficient information in order to determine what type of discharging mechanism is involved.

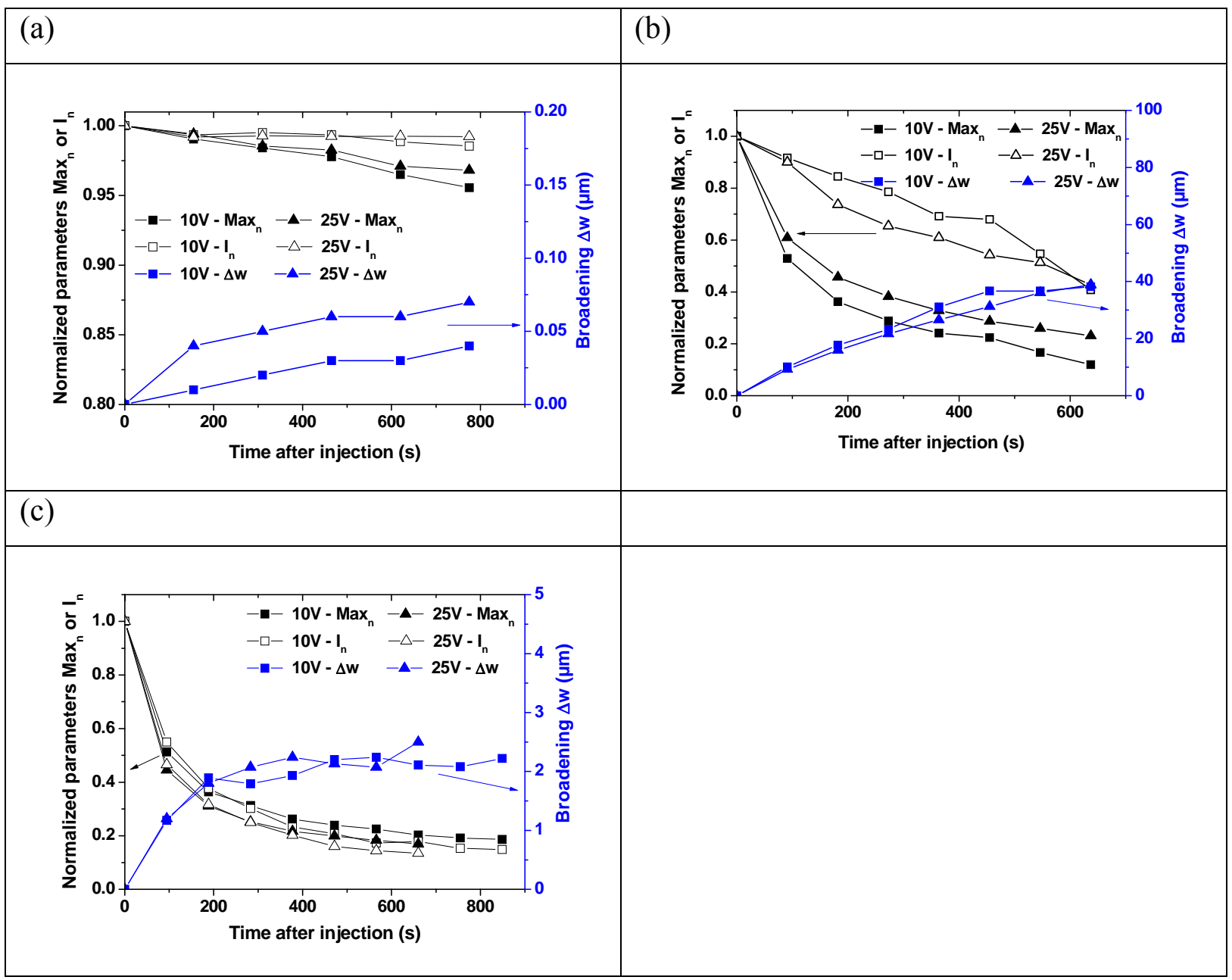

Figure 6: Comparison of evolution with time after injection of the normalized potential maximum, normalized integrated intensity $I_{n}$ and the peak broadening $\Delta w$ for (a) $\gamma=100$, (b) $\gamma=10$ and (c) $\gamma=5$. Bias injection is fixed to $10 \mathrm{~V}$ or $25 \mathrm{~V}$ during $2 \mathrm{~min}$.

For charge injection with negative bias no difference is observed in the potential decay with time for $\gamma=100$ and $\gamma=10$ layers. For $\gamma=5$-layer, two different mechanism are pointed out. 
Indeed, at early times the integrated intensity decreases slower compared to a positive bias, whereas at longer times it has the same decreasing dynamic regardless the polarity. Moreover, current measurements during charge injection show low conductivity for negative bias. So, the fast mechanism seems to be linked to carrier mobility and corresponds to hopping conductivity. But, further investigations are needed to accurately identify the involved decay mechanism.

\section{Conclusion}

Properties of a-SiON-H layers were tuned using process conditions. Thin layer characterization issue in term of structural and electrical properties is investigated. Adapted elipsometry procedure is presented to determine accurately film thickness. Moreover, results emphasize that KFM is powerful techniques to study charge/discharge phenomenon in dielectric film at nanoscale.

Results emphasize very different mechanisms for charges retention. So, process adjustment permits dielectric layer engineering in term of electrical properties. 
Appendix 1 - Some peculiarities of using spectroscopic ellipsometry to study thin dielectric layers deposited on Au-metalized Si-substrates

Spectroscopic ellipsometry (SE) is an optical characterization method which is based on the changes of initially polarized light after its reflection on a surface. It is largely applied to study the optical response of thin layers. SE is a non-destructive, low-cost diagnostic method and quite appropriate to follow (in-situ) and to control (in-situ and/or ex-situ) the thicknesses and the optical properties of deposited layers. However, it should be carefully performed in order to obtain correct interpretation of the recorded spectra. Here in the Appendix some critical points related to the interpretation procedure of SE recorded spectra are discussed. To properly apply the SE method in the current work, two challenges required to be overcome. The first challenge was related to the Au-metalized surface of the used Si substrates. The second one reflected actually thermal effects induced by the plasma deposition procedure.

According to the SE diagnostic method, the quantities recorded during measurements are: (i) $\tan (\Psi)$ which represents the ratio between moduli of the reflection coefficients, meaning that it is closely related to the changes in the amplitudes of the polarized electric field after reflection and (ii) $\cos (\Delta)$ that gives the phase difference $\left(\Delta=\delta_{p}-\delta_{s}\right)$, induced by the reflection, between the perpendicular $\left(\delta_{s}\right)$ and parallel $\left(\delta_{p}\right)$ components of the polarized electric field. Guided by the principles of SE on can see that incidence angle of the polarized light is strongly related to the sensitivity of SE method. The most accurate measurements would be performed with incidence angle that gives the largest phase shift $\left(\Delta_{\max }=\delta_{p}-\delta_{s}\right)$ between the parallel and the perpendicular components of the polarized electric field after reflection, i.e. for the incidence angle that returns $\cos (\Delta)=0$. Theoretically, it is the Brewster's angle. However, the Brewster's angle varies strongly as a function of the wavelength for Au. For example it is $68^{\circ}$ at $400 \mathrm{~nm}$, while it changes to $64^{\circ}$ at $500 \mathrm{~nm}$ and then it increases again up to $76^{\circ}$ at $700 \mathrm{~nm}^{27}$. Obviously a compromise for the incidence angle should be found in order to cover the whole spectral range between $250 \mathrm{~nm}$ and $850 \mathrm{~nm}$.

Typically the most appropriate incidence angle is determined after integration over the applied spectral range of $\cos (\Delta)$-variation for the full set of incidence angles (between $0^{\circ}$ and $90^{\circ}$ ). Quite often the range of accessible incidence angles is limited and the integration cannot be performed over the full range which prevents integration. It is either the case here. The panel on figure A1 represents $\cos (\Delta)$-variation for different incidence angles (from $60^{\circ}$ to $75^{\circ}$ )

\footnotetext{
${ }^{27} \mathrm{http}: / /$ refractiveindex.info
} 
as a function of the wavelength in the range $250-850 \mathrm{~nm}$ as recorded on the Au-layer used in the current work. In the range of strong irregular variation of Brewster's angle (400-700 nm), as show earlier, $\cos (\Delta)$ is close to 0 for incidence angles between $68^{\circ}$ and $70^{\circ}$. Our choice is $69^{\circ}$. The chosen incidence angle of $69^{\circ}$ can guarantee accuracy in the recorded spectra during SE measurements.

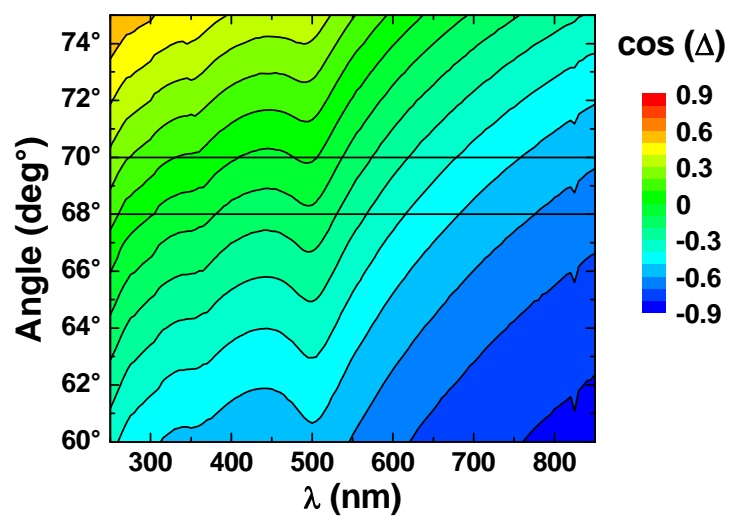

Figure A1: $\cos (\Delta)$-variation for different incidence angles as a function of the wavelength in the spectral range $250 \mathrm{~nm}-850 \mathrm{~nm}$ of the Au-layer used in this work.

The $\mathrm{Au} / \mathrm{Ti}$ metalized Si-substrate used in this work consists of Au-layer (260 nm) which is evaporated on Ti-layer (about $70 \mathrm{~nm}$ ) also evaporated in Si (100) substrate. The role of Ti-layer is to assure adhesion between the Au-layer and the Si-substrate. According to plasma deposition procedure applied for the elaboration of $\mathrm{a}-\mathrm{SiO}_{\mathrm{x}} \mathrm{N}_{\mathrm{y}}: \mathrm{H}$ layers the substrate is kept to $300^{\circ} \mathrm{C}$ during deposition. To guarantee the gas mixture, i.e. the used $\gamma$-value $\left(\gamma=\left[\mathrm{N}_{2} \mathrm{O}\right] /\left[\mathrm{SiH}_{4}\right]\right)$ in order to obtain repetitivity in the composition of deposited a-SiO $\mathrm{S}_{\mathrm{x}} \mathrm{N}_{\mathrm{y}}: \mathrm{H}-$ layers when varying $\gamma$-value $(\gamma=5,10,100)$ the plasma reactor is maintained under high vacuum ( $p=1.5 \times 10^{-6}$ Torr) during 16 hours. Simultaneously the substrate heating system is switched on to help the evacuation of all possible impurities, especially humidity, from the reactor chamber. It means that the $\mathrm{Au} / \mathrm{Ti}$ metalized Si-substrate is thermally treated (actually annealed) at $300^{\circ} \mathrm{C}$ during 16 hours. Then the a- $\mathrm{SiO}_{\mathrm{x}} \mathrm{N}_{\mathrm{y}}: \mathrm{H}$-layers deposition is performed. This procedure is respected for all the studied a- $\mathrm{SiO}_{\mathrm{x}} \mathrm{N}_{\mathrm{y}}: \mathrm{H}$ layers before their deposition. It gives the possibility to perform the assessment of the incidence angle for the SEmeasurements in order to establish the protocol minimizing the error-bars. The presented in panel A1 map of $\cos (\Delta)$-variation applies for annealed at $300^{\circ} \mathrm{C}$ under vacuum $\mathrm{Au} / \mathrm{Ti}$ metalized Si-substrate during 16 hours. 
Figure A2 represents $\tan (\Psi)$ and $\cos (\Delta)$ spectra of as-deposited Au-layer (black curve) and the same layer after annealing under vacuum at $300^{\circ} \mathrm{C}$ during 16 hours (red curve). One can notice from the obtained spectra (figure A2) of as-deposited Au-layer and of the same Aulayer, annealed at $300^{\circ} \mathrm{C}$ for $16 \mathrm{~h}$ that the properties of the Au-layes has been modified due to the thermal process. According to the $\tan (\Psi)$-variation as a function of the wavelength (figure A2(a)), the annealed Au-layer resembles massive gold.
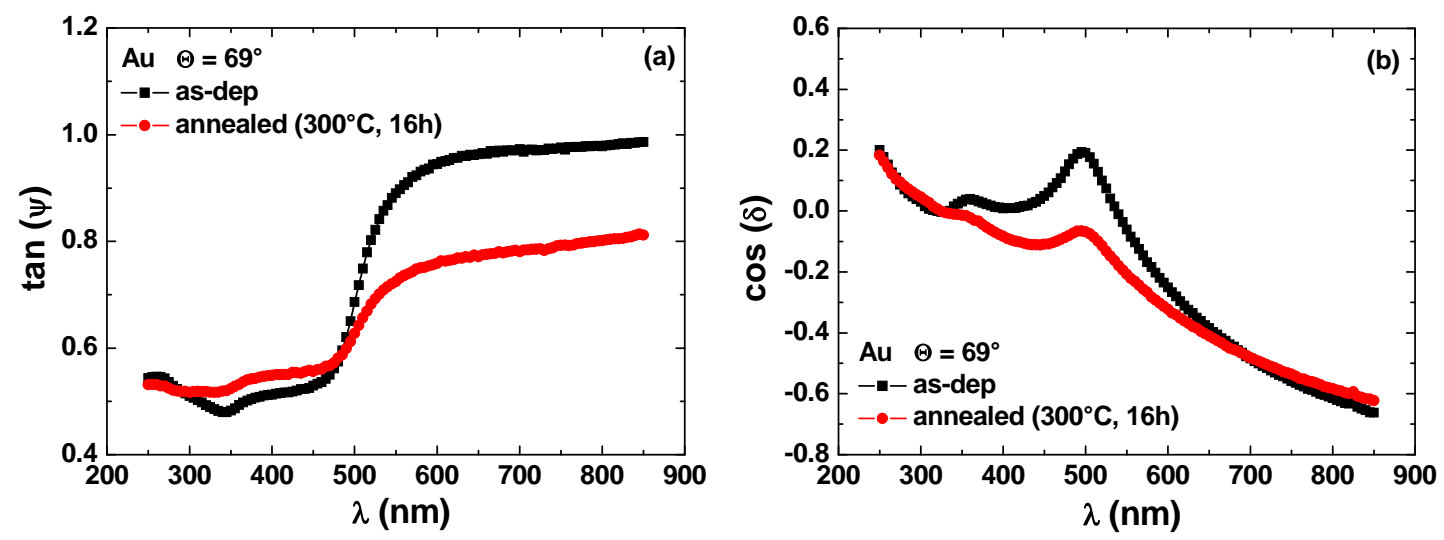

Figure A2: $\tan (\Psi)$ and $\cos (\Delta)$ spectra of as-deposited Au-layer (black curve) and the same layer after thermal treatment at $300^{\circ} \mathrm{C}$ during 16 hours (red curve).

The above observation is confirmed by the penetration depth of light in as-deposited and annealed Au-layers as shown in figure A3. The Au-layer with thickness like the deposited one $\left(d_{\mathrm{Au}}=260 \mathrm{~nm}\right)$ can be considered as substrate in the regression procedure during the spectra modeling following the SE measurements. It means that in the modeling procedure the considered structure will contain the Au-layer (as substrate), the plasma deposited a-SiO $\mathrm{x}_{\mathrm{y}}: \mathrm{H}$ layers and air (vacuum). The Si-substrate will be omitted.

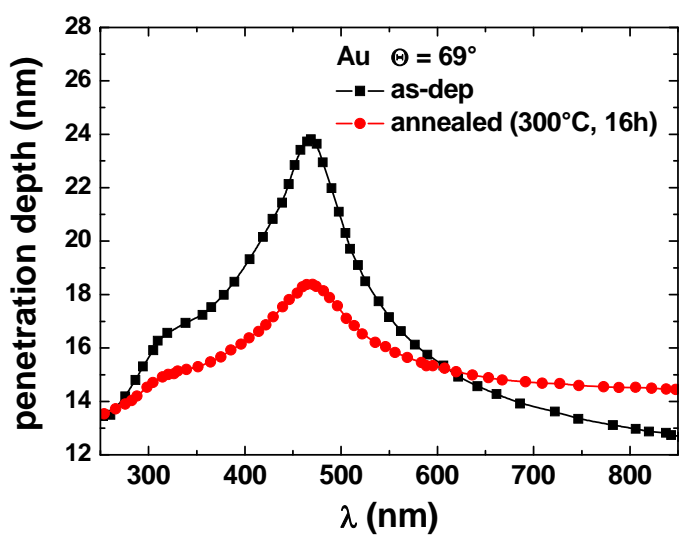

Figure A3: Light penetration depth of as-deposited Au-layer (black curve) and annealed Au-layer (red curve). 
The optical properties of Au-layer in the above determined structure (refractive index (n) and extinction coefficient $(\mathrm{k})$ ) as a function of the wavelength in the recorded spectral range should be specified during the SE-spectra modeling. Moreover the appropriate $n(\lambda)$ and $k(\lambda)$ must be used in the procedure when modeling the registered spectra. For the annealed Aulayer they can be extracted after bulk modeling of the recorded $\tan (\Psi)$ and $\cos (\Delta)$ spectra. Comparison between $\mathrm{n}(\lambda)$ and $\mathrm{k}(\lambda)$ of as-deposited and annealed Au-layer are shown in figure A4. It is clear that the thermal effects induced by the plasma deposition procedure modify the Au-layer.

When determine the a-SiO ${ }_{x} N_{y}: H-l a y e r$ thickness by using $n(\lambda)$ and $\mathrm{k}(\lambda)$ spectra of asdeposited Au-layer and not the correct ones of annealed Au-layer the obtained thickness value is higher. The difference is about $3.5 \mathrm{~nm}$ if the deposited layer is about $100 \mathrm{~nm}$ thick. It can increase if the $\mathrm{a}-\mathrm{SiO}_{\mathrm{x}} \mathrm{N}_{\mathrm{y}}: \mathrm{H}$ layer is thicker $(6.5 \mathrm{~nm}$ if the layer thickness is $320 \mathrm{~nm})$.
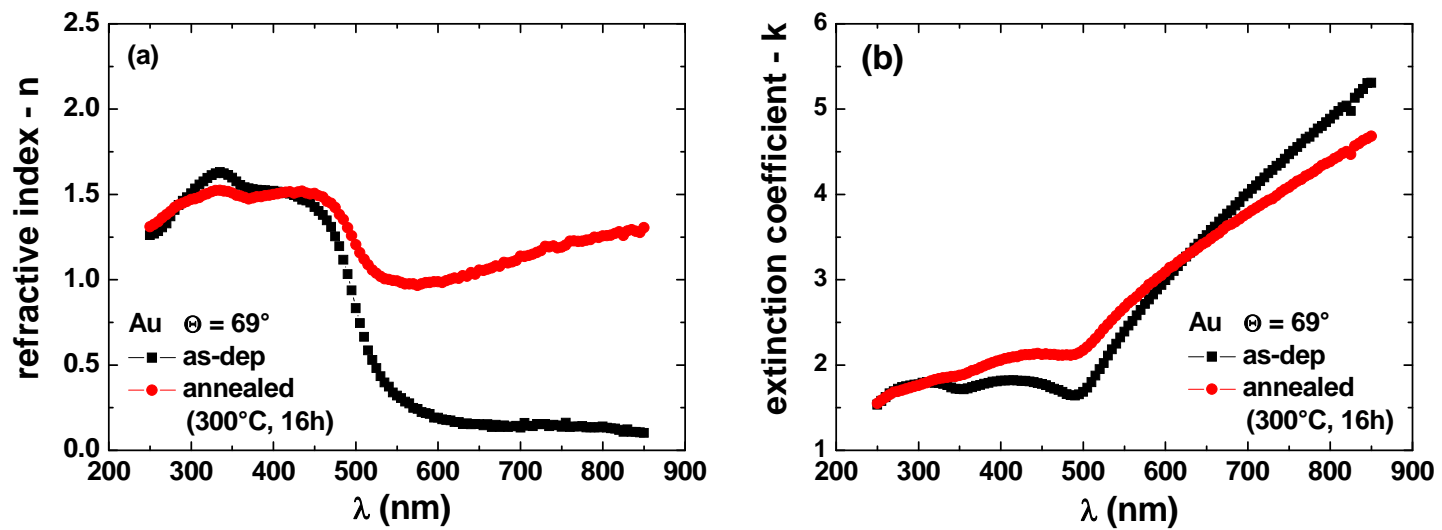

Figure A4: Refractive index on (a) and extinction coefficient on (b) of as-deposited Au-layer (black curve) and annealed Au-layer (red curve).

In conclusion, we can say that the spectroscopic ellipsometry is a very convenient diagnostic method to obtain the thicknesses and the optical properties of thin dielectric layers but its application should be carefully performed in order to limit the errors in the interpretation of the recorded spectra. 